\title{
Reconstructing MYC
}

\author{
David L. Levens ${ }^{1}$ \\ Gene Regulation Section, Laboratory of Pathology, National Cancer Institute, Center for Cancer Research, Bethesda, \\ Maryland 20892-1500, USA
}

\section{Who is MYC?}

The $c$-myc protooncogene is a senior administrator of the cell helping to allocate resources and direct proliferation, growth, differentiation, and apoptosis. As an oncogene, MYC is a high-ranking member of the gang of molecular thugs that hijacks cells and perpetrates cellular terror. We know a lot about MYC and its associates (Dang et al. 1999; Grandori et al. 2000; Boxer and Dang 2001; Eisenman 2001; Luscher 2001; Levens 2002; Lutz et al. 2002). The structural and biochemical features of the MYC family (MYC, N-MYC, and L-MYC) mark them as direct regulators of gene expression. As basic helix-loop-helix leucine zipper proteins (bHLH-ZIP), the MYCs acquire the capacity to bind the DNA sequence CACGTG (E-box) when dimerized with MAX (another bHLH-ZIP; McMahon et al. 2000; Bouchard et al. 2001). A head-to-tail pair of MYC-MAX dimers may, in turn, form a heterotetramer capable of bridging distant E-boxes (Nair and Burley 2003). MAX is titrated away from MYC by heterodimerizing with the competing MAD family members or with Mnt. All of these heterodimers also bind E-boxes, as do MAX homodimers. Each species of heterodimer associates with particular effector protein complexes to exert its influence on genomic targets. Among the broadly distributed positive enforcers of MYC action that are often recruited to target genes are chromatin remodeling (SWI/SNF relatives) and modifying complexes (TRAPP/GCN5 and relatives); these complexes mobilize nucleosomes and acetylate histones and/or other targets to activate gene expression (Cheng et al. 1999; McMahon et al. 2000; Luscher 2001). MYC binds TBP along an auxiliary pathway to control gene expression (Fig. 1). MAD and MNT generally oppose MYC action by enlisting histone deacetylase complexes (Fig. 1). Belying the terrifying organismal consequences of MYC misregulation, its influence on the expression of most target genes is only modest; MYC seldom exerts a dominating influence on natural or artificial targets.

Besides acting at the level of chromatin, MYC may also operate at later stages of the transcription cycle,

${ }^{1}$ Corresponding author.

E-MAIL levens@helix.nih.gov; FAX (301) 594-5227.

Article and publication are at http://www.genesdev.org/cgi/doi/10.1101/ gad.1095203. after pre-initiation complex formation (Eberhardy and Farnham 2001). In addition to using generic chromatin complexes to up- or down-regulate transcription, the MYC network also conscripts individual factors to modify expression locally on an ad hoc basis. For example, YY1, AP2, MIZ1, SP1, BRCA1, and other proteins interact directly with MYC, and so may directly modify the output of the MYC network (Fig. 1; Shrivastava et al. 1996; Sakamuro and Prendergast 1999).

\section{The far-flung reaches of the MYC web}

Identifying MYC targets at the RNA level once seemed the most straightforward approach to discover how MYC regulates cellular proliferation, growth, apoptosis, and differentiation. A variety of molecular, biological, and genetic approaches were devised to identify the mRNAs induced or (less often) repressed by MYC. It was expected, or at least hoped, that MYC action would be aimed at only a handful of primary targets with obvious links to the cell cycle, cell death, signal transduction, or cancer. Although a few of the first MYC targets identified seemed to be good candidates to participate in the strategic decisions of the cell, most of the genes that were recovered specified metabolic enzymes, structural proteins, and other proteins involved with the biochemical operation and assembly of the cell (for review, see Levens 2002). Because different groups of investigators using different approaches to identify MYC targets obtained different results, much discussion and debate concerned the physiological and pathological fidelity of the various systems used to enforce or block MYC action. Schemes to induce MYC targets using a MYC-estrogen receptor chimeric protein, virally expressed $\mathrm{MYC}$, other fusion proteins, comparisons of MYC knockout cells with MYC wild-type cells, and various strategies for supplying MYC encoded in plasmid or other vectors have all been exploited (Eilers et al. 1989; Mateyak et al. 1997; Hermeking et al. 2000). Approaches to interfere with MYC function have also been devised. Every scheme suffered from real or hypothetical caveats; among these were the inability to discriminate direct from indirect MYC targets, inappropriate targeting of genes due to artificially enforced high levels or nonphysiological levels of MYC, and alterations in mRNA levels of certain genes occurring as part of an adaptive physiological response to chronically high or low levels of MYC. 
Figure 1. The general properties of the MYC network are customized at individual binding sites. Heterodimers recruit a common set of effector proteins modulating chromatin activity and interact with TBP. These effector functions may also be modified by interactions with particular proteins that bind directly with MYC. MYC, in turn, may also modify the effector action of other proteins (e.g., neutralization of MIZ-1 activation). These sorts of interactions are likely to be controlled by the arrangement of the binding sites for those proteins recruited on an "ad hoc" basis. Lastly, other factors, recruited and arranged locally at promoters, may sculpt the action profile of the MYC network at each target. Green lines indicate positive effector function; red lines indicate negative effector function. The broken line represents a functionally uncharacterized in vivo interaction with DRE reported in this issue (Orian et al. 2003).

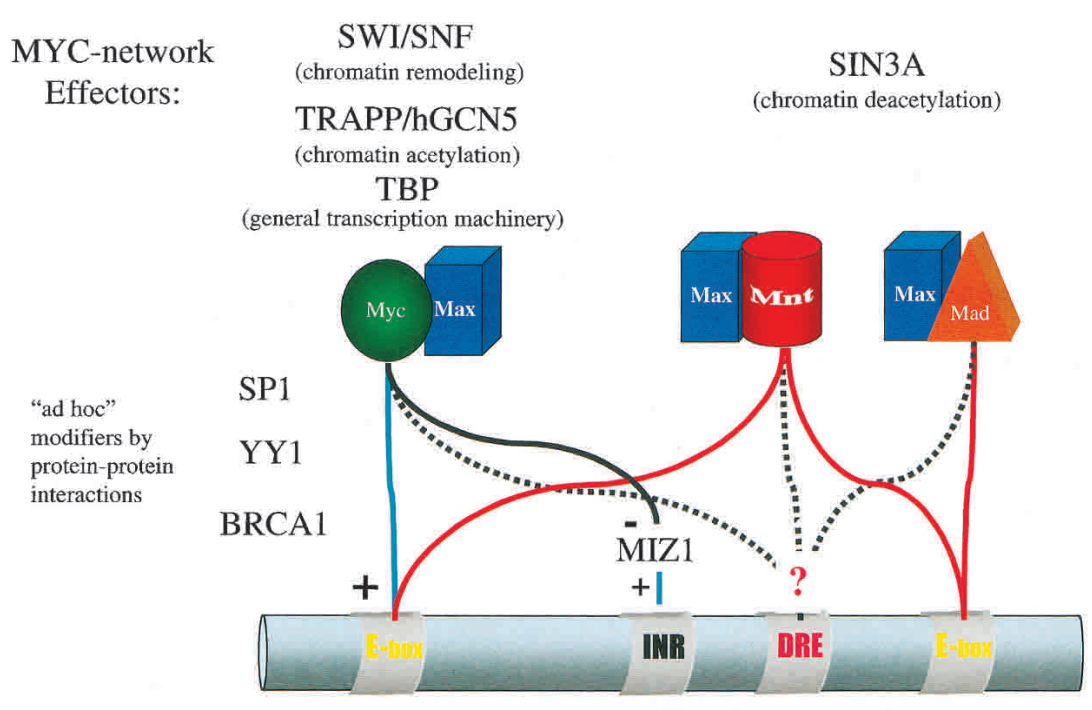

Promoter/enhancer context/location specific modifiers of MYC-network activity
Global analysis of gene expression has failed to expose the workings of the MYC network. A variety of cDNA microarray and SAGE experiments have yielded a large number of provisional MYC targets. Although there is significant overlap among the MYCregulated genes identified, considerable differences also occur. It has been impossible to decipher which MYC targets are key to understanding MYC function and pathology and which may be of secondary importance or irrelevant.

\section{Where does MYC act?}

To modify the expression of a target gene, MYC must first be recruited there (ignoring fanciful schemes for action at a distance). With the availability of the complete sequence of the human genome as well as the genomes of several important model organisms, all of the E-boxes in a genome may be rapidly collated in silico with nearby known genes, ORFs, and ESTs. If MYC-binding sites were recognizable a priori, sifting candidate MYC target cDNA microarray data against genomic sequences would rapidly yield direct MYC targets. But is an in silico E-box necessarily an in vivo E-box? How much physiology and pathology of the MYC web may be elucidated by such an analysis? Two studies described in this issue of Genes \& Development (Fernandez et al. 2003; Orian et al. 2003) used different approaches to find the sequences that bind MYC in vivo and to relate them to MYC targets. The authors of both studies conclude that MYC binds and directly influences the expression of about $10 \%$ of the genes in flies and in us.

Amati and colleagues (Fernandez et al. 2003) scanned genomic DNA sequences for E-boxes in proximity to transcription start sites and systematically applied conventional chromatin immunoprecipitation (ChIP) assays at 809 sites in the human genome. The recovery of pro- moter fragments relative to unselected genomic DNA was assessed by real-time PCR; the fractional occupancy of MYC at different sites was reasonably assumed to be proportional to this RT-PCR signal. As MYC levels were increased in different cell systems, up to $85 \%$ of promoter-associated E-boxes were bound. Extrapolating from their data set, Amati and colleagues (Fernandex et al. 2003) estimated that up to $9 \%$ of genes are MYC targets at physiological levels of MYC (but since no normal cells, activated or resting, were included in this study, the estimate of physiological binding is an extrapolation), and that even this large fraction is increased with supraphysiological concentrations of MYC. E-box occupancy was related to several parameters including (1) MYC levels, (2) distance from the start site of transcription (occupancy declined with increasing distance), and (3) proximity to CpG islands, although this parameter is related to distance from the start site. Binding to noncanonical E-boxes was not examined, nor was recruitment of MYC to promoters via protein-protein interactions explored. Taking both of these considerations into account would increase the number of MYC targets in vivo. Relative occupancy of E-boxes at promoters did not predict the magnitude of mRNA induction by MYC. Some highly MYC-activated genes were weakly bound, whereas other marginally induced targets exhibited strong binding. Sorting MYC targets according to the fractional occupancy of their E-boxes revealed that the relative ranking of the individual binding sites was conserved between several cellular systems, with few exceptions. The existence of some exceptions, however, shows that cell type or physiological factors may override locally the influence of MYC at certain promoters. Because MYC recruits acetylase complexes, the degree of histone $\mathrm{H} 3$ and $\mathrm{H} 4$ acetylation at MYC target loci was also evaluated by ChIP and real-time PCR. Although MYC binding was qualitatively related to histone acetylation 
in a binary "yes-no" fashion, at individual promoters, MYC binding did not quantitatively predict the extent of acetylation. MYC targets generally had higher levels of basal acetylation, perhaps reflecting constitutive housekeeping expression; this basal activity may have obscured the correlation between MYC binding and histone modification. E-boxes unlinked to genes exhibited only very low levels of MYC binding.

A separate study by Orian et al. (2003) used a new approach to covalently tag the DNA at MYC-network target loci in vivo (van Steensel et al. 2001). By expressing a fusion protein of the prokaryotic Escherichia coli Dam methylase with MYC (or MAD or MNT) in Drosophila cells, those investigators generated proteins that while tethered at E-boxes or other sites of MYC recruitment, would methylate local GATC sequences encountered through DNA looping. Cleavage of the genomic DNA recovered from these cells with the methylationrequiring Dpn I restriction enzyme enriched MYC targets as small fragments from otherwise high-molecularweight DNA. Interrogating Drosophila cDNA microarrays with these sequences identified genes targeted by the dMYC network. Offsetting the disadvantage of requiring a chimeric protein, this nonbiased approach identifies MYC bound to non-E-box targets, bound via protein-protein interactions or bound at sites that engage MYC only transiently, if not fleetingly. Having identified dMYC targets, those investigators next queried the Drosophila genome with the computer program REDUCE to extract shared sequence motifs (Bussemaker et al. 2001). (Although REDUCE was originally developed to associate sequence motifs with expression data, the more direct linkage between sequence motifs and binding as assessed with MYC-Dam chimera methylation is even more amenable to this analysis.) As MYC-Dam expression was driven to higher levels, the number of targets recovered also increased, reaching as high as $15 \%$ of Drosophila genes! Considering that only $40 \%$ of these targets bore canonical E-boxes, this result is in good agreement with the estimate by Fernandez et al. (2003) that $6 \%-9 \%$ of genes bind MYC through E-boxes. The DRE sequence, a motif statistically associated with cell cycle-regulated genes, was also highly associated with MYC. There is no evidence that MYC directly recognizes this element, so it seems likely that MYC is recruited indirectly to DRE targets through protein-protein interactions. Such interactions may also help to stabilize dMYC-DNA complexes at suboptimal sites. Using similar Dam chimeras, Orian and colleagues (Orian et al. 2003) explored the genomic dominion of the entire MYC-MAX-Mnt network. Each heterodimer claimed a characteristic set of targets, but with considerable overlap. (The lack of full concordance indicates that each player may bind with uncharacterized partners at noncanonical sites.) The binding spectrum of MYC with genomic DNA only coarsely mirrored the response of MYC target RNA, yielding a binary on-off relationship, but not a quantitative correlation between binding strength and expression levels.

\section{What does MYC do?}

There are many MYC targets, as identified by binding or by expression analysis, and probably even more exist, because nonmessenger RNAs transcribed by RNA polymerase III have very recently been discovered to bind and be up-regulated by MYC (Gomez-Roman et al. 2003). MYC modifies the expression of all sorts of genes involved with most major cellular processes. Many of these targets are basally expressed, and so it appears that MYC adjusts the level of diverse targets, perhaps helping to integrate the flux of metabolites and information through various pathways. Just as changes in the level and activity of the CAP/CRP protein reverberate through the E. coli cell (regulating $5 \%-10 \%$ of proteins) to coordinate cell growth and metabolism, so MYC may have been selected to serve such an administrative role (Kolb et al. 1993; Thieffry et al. 1998). Adjustments in the supply and distribution of the biochemical resources feeding cell growth and proliferation undoubtedly contribute to proper cellular and organismal homeostasis. Although the information and metabolic networks of a cell are remarkably robust with respect to viability, perturbing the operation of some cellular subsystems would be expected to modify the flux of materials and information through linked subsystems, altering physiology or contributing to pathology (Ibarra et al. 2002; Stelling et al. 2002). Misexpression of MYC may not elicit the catastrophic failure of a single, crucial nexus, but by the incremental maladjustment of dozens, if not hundreds of targets, various metabolic and biochemical functions may be driven into a zone of chaotic instability. For example, overexpressing the MYC target serine hydroxymethyltransferase accelerates proliferation; concurrent effects on other pathways ultimately bypass systems restraining proliferation (Nikiforov et al. 2002). It is certainly possible, however, that the misregulation of only a few key targets that drive oncogenesis is obscured by a background of irrelevant changes in gene expression. According to this scenario, there maybe no particular "logic" relating MYC targets; rather the MYC network is just a set of transcription factors that happens to overdrive a bad combination of targets. Other transcription factors regulating a similarly broad range of targets may by circumstance do the same.

\section{What is a true MYC target?}

Is it a binding site or an mRNA regulated by MYC? Applying only one definition risks amassing a catalog of genes where MYC may bind, but exert only marginal influence on expression, but application of criteria that are too stringent may obscure important targets where even small deviations in expression are biologically important or where the influence of MYC is modified by other parameters (Watson et al. 2002). Some scheme must relate the physical presence of MYC at a gene with the quantitative output of its promoter, and some rationale (or at least a rationalization) must account for the apparent discrepancies between MYC-binding and MYC- 
network-regulated expression. There is really no reason to expect a very tight correlation between steady-state binding and activity. Too many parameters conspire to degrade this correlation. For example, MYC need not be stably bound at target sites to influence transcription. At some sites MYC may act through a hit-and-run mechanism consistent with the nomadic behavior of other transcription factors, dynamically binding and moving on. Indeed at one important target, hTERT, it seems likely that MYC evacuates the promoter, probably before promoter escape. An E-box at +22 of the hTERT promoter has been shown to bind MYC and activate transcription (Wang et al. 1998; Greenberg et al. 1999; Horikawa et al. 1999; Oh et al. 2001; Veldman et al. 2001). It is difficult to envision a pre-initiation complex (PIC), fully accoutered with the several dozens of polypeptides comprising RNA polymerase II, TFIID, mediator, TFIIH, etc., cohabitating this promoter with the closely tethered multisubunit MYC/coactivator complex. It is almost inconceivable that the PIC could transition to promoter escape without evicting the MYC complex. If MYC interacts with its targets (at least some of them) only transiently prior to PIC formation or promoter escape, the pol II-MYC co-occupancy problem vanishes; however, this arrangement precludes full E-box occupancy and so diminishes the quantitative correlation between binding and mRNA levels. In principle this correlation might also be weakened if formaldehyde crosslinking were to trap MYC transiently bound at irrelevant sites. Since every sequence-specific DNA-binding protein is in some measure also a nonspecific binder, MYC must explore many non-E-box sequences before settling at a proper site. Exactly this behavior has been proven in vivo for the homeobox proteins Eve and Ftz; moreover, these proteins may contribute to gene activity even when bound to nonspecific (or at least highly noncanonical) sites (Liang and Biggin 1998). However, Fernandez et al. (2003) failed to find MYC associating with nonpromoter E-boxes. Nonspecific binding was also not a problem in the study by Orian et al. (2003), where the release of a DNA fragment with the MYC-Dam methylase chimera demands four modifications of the target DNA (both strands at two sites). The completion of these modifications probably requires more than a single fleeting, spurious interaction. If the complex relationship between MYC-binding sites and target gene expression is not a technical artefact, then other variables must modify the effector activity of MYC.

\section{Location, location, location}

The effector function of a DNA or chromatin-bound regulatory protein is likely to be modified by the presence and arrangement of other regulatory elements at any gene. There may be no prototypical response to MYC; every promoter may have its own personality. The number of E-boxes and their locations relative to the start site of transcription are not stereotyped. Flexibility in the numbers and positions of E-boxes is likely to be an important parameter contributing to the characteristic activity profile of each MYC target. E-boxes that overlap with the binding sites for other factors would force the MYC network to compete with its prospective neighbor for binding in order to influence target expression; who wins would depend on the abundance and activity of MYC and its rivals. A cell type- or situation-specific neighbor would restrict target expression only in particular settings. Binding to DNA does not complete MYC action, and so different promoter-bound factors could interfere with the MYC network subsequent to DNA binding, and this interference is likely to be highly idiosyncratic. What might happen when MYC recruits a histone acetylase while another factor recruits a histone deacetylase? What if MYC, often a weak activator, displaces a more potent activator?

Positive MYC action is likely to be context-specific. At some promoters, MYC may cooperate with other activators in the synergistic and cooperative construction of an enhanceosome-like coactivator landing site (Thanos and Maniatis 1995). In this case, MYC action would be inextricably coupled with the abundance and activity of its partners. At other promoters, where each transcription factor acts as a free agent, the influence of MYC may be augmented or diluted incrementally by the action of other players.

Multiple E-boxes increase the likelihood of recruiting multiple MYC-network complexes to a gene. Multiple binding sites for MYC, as for most transcription factors, leads to synergistic activation (Bellofernandez et al. 1993). Even if E-boxes are only transiently occupied, extra E-boxes increase the probability of finding a MYC complex at a gene at any instant. MYC-MAX and MADMAX are sometimes tetrameric and when so are bivalent for DNA binding. Multiple E-boxes would allow the tetramer to toggle between sites. Considering that some E-boxes are found downstream of start sites, often in intron 1, toggling would enable MYC complexes to leapfrog over elongating transcription complexes while remaining DNA-bound. Such molecular acrobatics would be heavily influenced by the architectural constraints of DNA bending, helical phase, and chromatin structure at and between the interacting sites. Similar gymnastics will influence the collaboration of MYC complexes with other DNA-bound transcription factors to create a surface that grabs the proper coactivator, corepressor, or chromatin remodeling complexes. In this situation, the activity of MYC and its DNA-binding partners would synergize in a locus-specific manner. The details describing the number and arrangement of E-boxes as well as MYC-cooperating or -antagonizing elements may well dictate the responses of otherwise similar genes; all Eboxes are not equivalent (Boyd et al. 1998).

\section{How much MYC?}

Freshman chemistry works even in vivo. The fractional occupancy at many, if not most, MYC-binding sites will be largely determined by mass action, and so will be proportional to the concentration of free MYC and its partners. Hence, figuring out how much of each MYC- 
network member to make is an important cellular calculation. For MYC production, this arithmetic is reckoned at the levels of transcription, RNA processing, RNA stability, translation, and MYC turnover. The $c$ myc promoter has been found to bind a complex and a still incompletely enumerated set of transcription factors. The logic of this diversity almost certainly derives from feedback coupling of $c$-myc transcription with the functional status of most (if not all) of the subsystems assembled from or reliant upon the targets of the MYC network. There is no well-defined $c$-myc enhancer or locus control region, and there is no evidence for the assembly of a single functioning enhanceosome such as the one that occurs upon the $\beta$-interferon gene. Thus the $c$-myc promoter may serve as a processor of diverse inputs to set $c$-myc mRNA output. These multiple positive and negative inputs may act simultaneously or sequentially during PIC assembly, or exert their influence postinitiation during the progression to promoter escape regulating the holdback or release of the RNA polymerase paused at the MYC promoter under most circumstances (Liu et al. 2000, 2001). Probably no single algorithm accounts for the complex functioning of the $c$-myc promoter. The rules for integrating the multitude of DNA- and chromatin-bound factors bound to the $c-m y c$ gene are not known and are highly context-dependent. Chromatin boundaries imposed by factors such as CTCF may include or limit the activity of cis-elements associated with the $c-m y c$ gene (Filippova et al. 1996). The same factors that exert dominating influence on $c$-myc transcription in one biological setting may be feeble or impotent under other circumstances. $c$-myc mRNA turnover is regulated and coordinated with translation (Lemm and Ross 2002). Binding of auxiliary factors to the coding region of $c-m y c$ mRNA is required to impose this control. The 3 '-UTR of MYC may contribute to RNA stability in some situations, but not others (Aghib and Bishop 1991; Langa et al. 2001). MYC protein turnover is also regulated, and there are different subcellular pools of MYC in cells (Sears et al. 2000; Tworkowski et al. 2002). The functional roles of these different pools have not yet been defined, but such subcellular specialization would seem likely to have biological consequences. The interplay between the transcriptional, posttranscriptional, translational, and posttranslational regulation of MYC has not been elucidated. It is not known whether these layers always act to reinforce each other or whether these systems may sometimes oppose each other to dampen fluctuations in MYC levels. To clamp a factor at constant activity in an environment where information, building materials, and nutrients are in temporal or spatial flux may require more complicated feedback mechanisms than "on-or-off." Less is known about the promoters and regulation of other MYC-network members. max mRNA expression is more invariant than that of $c-m y c$, but MAX protein levels are regulated (Peters et al. 1997). MAD is up-regulated during differentiation, consistent with its role as a transcriptional repressor and with the shutoff of MYC action during differentiation (Grandori et al. 2000).

\section{Reconstructing MYC}

Despite the daunting complexity of the MYC system, the explication of the patterns, if not mechanisms of its operation, may not be far off. To reconstruct the MYC web, we will need to recognize the context-dependent rules and patterns governing MYC/MAX/MAD/MNT action. The number of significant variations of the location of MYC-binding sites, the identity of neighboring cis-elements, and the nature of MYC partners, although large, is nevertheless finite. Through its large number of targets, the MYC network extends a global reach into all cellular processes. This daunting complexity also affords opportunities to develop and screen hypotheses a posteriora to sort and subclassify MYC-network targets. Comparison of different biological and cellular systems, under various conditions, will trace the connections of the MYC network through the densely entangled metabolic, regulatory, and structural circuitry of the cell. Recognizing and proving these connections will require the interweaving of genome-wide and gene-specific experiments. Flexible computational methods that sort, rank, and classify expression data with genome-wide binding data are increasing in number and power (there are far too many publications describing computational methods of locating regulatory elements to cite in this article). As these programs become accessible and user-friendly, they will be evaluated and proofed at the lab bench. Exploration of the combinatorial usage of cis-elements considering their numbers, position, and order will help to develop the grammar and syntax of the regulatory language spoken by MYC. Computational methods filter data sets against spatial constraints such as distance (between various elements, from transcription start sites, insulators, origins of replication, nearby genes, response to torsional stress, etc.) and other attributes of DNA sequence (i.e., G-C islands, repetitive elements, A-tracts that bend DNA, helical phase relationships, etc.). These analyses will likely expose patterns defining subsets of coregulated MYC targets. Subtle changes in the regulatory topography of a MYC target promoter will customize its action profile, just as occurs with the $\beta$-interferon enhanceosome (Lomvardas and Thanos 2002). The large numbers of MYC targets participate in many pathways with different kinetics and different dynamic ranges. Using formidable computing methods, elegant experiments, imagination, and brute force, the nodes in the MYC web will be exposed. Systematically reconstructing the MYC-protooncogene network may expose links that can be therapeutically manipulated to compel the rehabilitation of its homicidal alter ego, the MYC oncoprotein.

\section{Acknowledgments}

I thank Drs. Lance Liotta and Susan Mackem for critical review and Dr. Robert Larossa for advice.

\section{References}

Aghib, D.F. and Bishop, J.M. 1991. A 3' truncation of Myc caused by chromosomal translocation in a human T-cell leu- 
kemia is tumorigenic when tested in established rat fibroblasts. Oncogene 6: 2371-2375.

Bellofernandez, C., Packham, G., and Cleveland, J.L. 1993. The ornithine decarboxylase gene is a transcriptional target of C-Myc. Proc. Natl. Acad. Sci. 90: 7804-7808.

Bouchard, C., Dittrich, O., Kiermaier, A., Dohmann, K., Menkel, A., Eilers, M., and Luscher, B. 2001. Regulation of cyclin D2 gene expression by the Myc/Max/Mad network: Mycdependent TRRAP recruitment and histone acetylation at the cyclin D2 promoter. Genes \& Dev. 15: 2042-2047.

Boxer, L.M. and Dang, C.V. 2001. Translocations involving cmyc and c-myc function. Oncogene 20: 5595-5610.

Boyd, K.E., Wells, J., Gutman, J., Bartley, S.M., and Farnham, P.J. 1998. c-Myc target gene specificity is determined by a postDNA-binding mechanism. Proc. Natl. Acad. Sci. 95: 1388713892.

Bussemaker, H.J., Li, H., and Siggia, E.D. 2001. Regulatory element detection using correlation with expression. Nat. Genet. 27: 167-171.

Cheng, S.W., Davies, K.P., Yung, E., Beltran, R.J., Yu, J., and Kalpana, G.V. 1999. c-MYC interacts with INI1/hSNF5 and requires the SWI/SNF complex for transactivation function. Nat. Genet. 22: 102-105.

Dang, C.V., Resar, L.M.S., Emison, E., Kim, S., Li, Q., Prescott, J.E., Wonsey, D., and Zeller, K. 1999. Function of the c-Myc oncogenic transcription factor. Exp. Cell Res. 253: 63-77.

Eberhardy, S.R. and Farnham, P.J. 2001. c-Myc mediates activation of the cad promoter via a post-RNA polymerase II recruitment mechanism. J. Biol. Chem. 276: 48562-48571.

Eilers, M., Picard, D., Yamamoto, K.R., and Bishop, J.M. 1989. Chimeras of Myc oncoprotein and steroid-receptors cause hormone-dependent transformation of cells. Nature 340: 6668.

Eisenman, R.N. 2001. Deconstructing Myc. Genes \& Dev. 15: 2023-2030

Fernandez, P.C., Frank, S.R., Wang, L., Schroeder, M., Liu, S., Greene, J., Cocito, A., and Amati, B. 2003. Genomic targets of the human c-Myc protein. Genes \& Dev. (this issue).

Filippova, G.N., Fagerlie, S., Klenova, E.M., Myers, C., Dehner, Y., Goodwin, G., Neiman, P.E., Collins, S.J., and Lobanenkov, V.V. 1996. An exceptionally conserved transcriptional repressor, CTCF, employs different combinations of zinc fingers to bind diverged promoter sequences of avian and mammalian c-myc oncogenes. Mol. Cell Biol. 16: 2802-2813.

Gomez-Roman, N., Grandori, C., Eisenman, R.N., and White, R.J. 2003. Direct activation of RNA polymerase III transcription by c-Myc. Nature 421: 290-294.

Grandori, C., Cowley, S.M., James, L.P., and Eisenman, R.N. 2000. The Myc/Max/Mad network and the transcriptional control of cell behavior. Annu. Rev. Cell Dev. Biol. 16: 653699.

Greenberg, R.A., O'Hagan, R.C., Deng, H., Xiao, Q., Hann, S.R., Adams, R.R., Lichtsteiner, S., Chin, L., Morin, G.B., and DePinho, R.A. 1999. Telomerase reverse transcriptase gene is a direct target of c-Myc but is not functionally equivalent in cellular transformation. Oncogene 18: 1219-1226.

Hermeking, H., Rago, C., Schuhmacher, M., Li, Q., Barrett, J.F., Obaya, A.J., O'Connell, B.C., Mateyak, M.K., Tam, W., Kohlhuber, F., et al. 2000. Identification of CDK4 as a target of c-MYC. Proc. Natl. Acad. Sci. 97: 2229-2234.

Horikawa, I., Cable, P.L., Afshari, C., and Barrett, J.C. 1999. Cloning and characterization of the promoter region of human telomerase reverse transcriptase gene. Cancer Res. 59: $826-830$.

Ibarra, R.U., Edwards, J.S., and Palsson, B.O. 2002. Escherichia coli K-12 undergoes adaptive evolution to achieve in silico predicted optimal growth. Nature 420: 186-189.

Kolb, A., Busby, S., Buc, H., Garges, S., and Adhya, S. 1993. Transcriptional regulation by cAMP and its receptor protein. Annu. Rev. Biochem. 62: 749-795.

Langa, F., Lafon, I., Vandormael-Pournin, S., Vidaud, M., Babinet, C., and Morello, D. 2001. Healthy mice with an altered c-myc gene: Role of the 3' untranslated region revisited. Oncogene 20: 4344-4353.

Lemm, I. and Ross, J. 2002. Regulation of c-myc mRNA decay by translational pausing in a coding region instability determinant. Mol. Cell Biol. 22: 3959-3969.

Levens, D. 2002. Disentangling the MYC web. Proc. Nat1. Acad. Sci. 99: 5757-5759.

Liang, Z. and Biggin, M.D. 1998. Eve and ftz regulate a wide array of genes in blastoderm embryos: The selector homeoproteins directly or indirectly regulate most genes in Drosophila. Development 125: 4471-4482.

Liu, J.H., He, L.S., Collins, I., Ge, H., Libutti, D., Li, J.F., Egly, J.M., and Levens, D. 2000. The FBP interacting repressor targets TFIIH to inhibit activated transcription. Mol. Cell 5: $331-341$.

Liu, J.H., Akoulitchev, S., Weber, A., Ge, H., Chuikov, S., Libutti, D., Wang, X.W., Conaway, J.W., Harris, C.C., Conaway, R.C., et al. 2001. Defective interplay of activators and repressors with TFIH in xeroderma pigmentosum. Cell 104: 353-363.

Lomvardas, S. and Thanos, D. 2002. Modifying gene expression programs by altering core promoter chromatin architecture. Cell 110: 261-271.

Luscher, B. 2001. Function and regulation of the transcription factors of the Myc/Max/Mad network. Gene 277: 1-14.

Lutz, W., Leon, J., and Eilers, M. 2002. Contributions of Myc to tumorigenesis. Biochim. Biophys. Acta 1602: 61-71.

Mateyak, M.K., Obaya, A.J., Adachi, S., and Sedivy, J.M. 1997. Phenotypes of c-myc-deficient rat fibroblasts isolated by targeted homologous recombination. Cell Growth Differ. 8: 1039-1048.

McMahon, S.B., Wood, M.A., and Cole, M.D. 2000. The essential cofactor TRRAP recruits the histone acetyltransferase hGCN5 to c-Myc. Mol. Cell. Biol. 20: 556-562.

Nair, S.K. and Burley, S.K. 2003. X-ray structures of Myc-Max and Mad-Max recognizing DNA. Molecular bases of regulation by proto-oncogenic transcription factors. Cell 112: 193-205.

Nikiforov, M.A., Chandriani, S., O'Connell, B., Petrenko, O., Kotenko, I., Beavis, A., Sedivy, J.M., and Cole, M.D. 2002. A functional screen for Myc-responsive genes reveals serine hydroxymethyltransferase, a major source of the one-carbon unit for cell metabolism. Mol. Cell Biol. 22: 5793-5800.

Oh, S.T., Kyo, S., and Laimins, L.A. 2001. Telomerase activation by human papillomavirus type $16 \mathrm{E} 6$ protein: Induction of human telomerase reverse transcriptase expression through Myc and GC-rich Sp1 binding sites. J. Virol. 75: 5559-5566.

Orian, A., van Steensel, B., Delrow, J., Bussemaker, H.J., Li, L., Sawado, T., Williams, E., Loo, L.W.M., Cowley, S.M., Yost, C., et al. 2003. Genomic binding by the Drosophila Myc, Max, Mad/Mnt transcription factor network. Genes \& Dev. (this issue).

Peters, M.A., Sollenberger, K.G., Kao, T.L., and Taparowsky, E.J. 1997. A minimal regulatory region maintains constitutive expression of the max gene. Mol. Cell. Biol. 17: 1037-1048.

Sakamuro, D. and Prendergast, G.C. 1999. New Myc-interacting proteins: A second Myc network emerges. Oncogene 18: $2942-2954$.

Sears, R., Nuckolls, F., Haura, E., Taya, Y., Tamai, K., and Nevins, J.R. 2000. Multiple Ras-dependent phosphorylation pathways regulate Myc protein stability. Genes \& Dev. 
14: $2501-2514$.

Shrivastava, A., Yu, J., Artandi, S., and Calame, K. 1996. YY1 and c-Myc associate in vivo in a manner that depends on c-Myc levels. Proc. Natl. Acad. Sci. 93: 10638-10641.

Stelling, J., Klamt, S., Bettenbrock, K., Schuster, S., and Gilles, E.D. 2002. Metabolic network structure determines key aspects of functionality and regulation. Nature 420: 190-193.

Thanos, D. and Maniatis, T. 1995. Virus induction of human IFN $\beta$ gene expression requires the assembly of an enhanceosome. Cell 83: 1091-1100.

Thieffry, D., Salgado, H., Huerta, A.M., and Collado-Vides, J. 1998. Prediction of transcriptional regulatory sites in the complete genome sequence of Escherichia coli K-12. Bioinformatics 14: 391-400.

Tworkowski, K.A., Salghetti, S.E., and Tansey, W.P. 2002. Stable and unstable pools of Myc protein exist in human cells. Oncogene 21: 8515-8520.

van Steensel, B., Delrow, J., and Henikoff, S. 2001. Chromatin profiling using targeted DNA adenine methyltransferase. Nat. Genet. 27: 304-308.

Veldman, T., Horikawa, I., Barrett, J.C., and Schlegel, R. 2001. Transcriptional activation of the telomerase hTERT gene by human papillomavirus type 16 E6 oncoprotein. J. Virol. 75: 4467-4472.

Wang, J., Xie, L.Y., Allan, S., Beach, D., and Hannon, G.J. 1998. Myc activates telomerase. Genes \& Dev. 12: 1769-1774.

Watson, J.D., Oster, S.K., Shago, M., Khosravi, F., and Penn, L.Z. 2002. Identifying genes regulated in a Myc-dependent manner. J. Biol. Chem. 277: 36921-36930. 


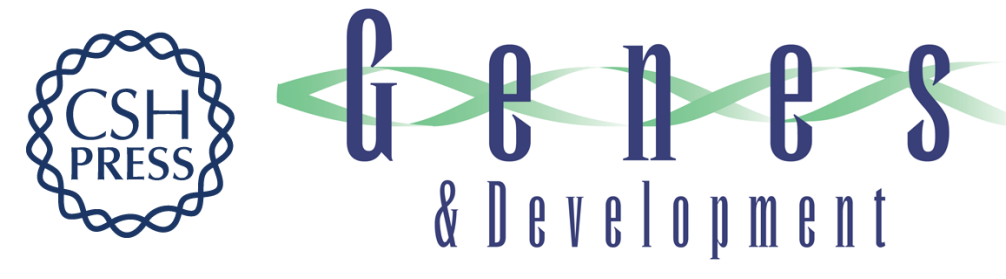

\section{Reconstructing MYC}

David L. Levens

Genes Dev. 2003, 17:

Access the most recent version at doi:10.1101/gad.1095203

References This article cites 45 articles, 21 of which can be accessed free at: http://genesdev.cshlp.org/content/17/9/1071.full.html\#ref-list-1

License

Email Alerting Receive free email alerts when new articles cite this article - sign up in the box at the top Service right corner of the article or click here.

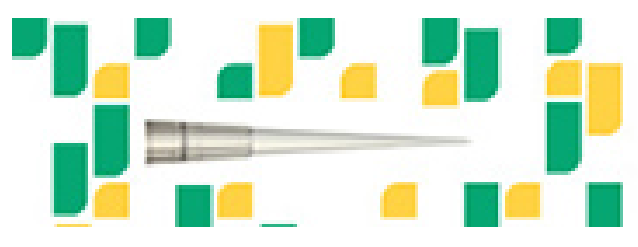

Focused on your science. 